\title{
Creación de capacidades para el desarrollo de liderazgo a los cirujanos ortopédicos en América Latina
}

\author{
Building leadership development capacity for orthopaedic surgeons in Latin America \\ Madeline C MacKechnie, ${ }^{*}$ Luis G Padilla Rojas, ${ }^{\ddagger}$ Fernando De la Huerta, $§$ Leonardo López Almejo," \\ José Máximo Gómez Acevedo," Sergio Quintero Hernández, ** Theodore Miclau, * \\ Grupo de Programa de Desarrollo de Liderazgo ${ }^{\ddagger \ddagger}$ \\ *Orthopaedic Trauma Institute, Department of Orthopaedic Surgery, University of California, San Francisco. Zuckerberg San \\ Francisco General Hospital; ¥ Hospital Puerta de Hierro, Zapopan, México; \&Unidad de Ortopedia y Traumatología, Guadalajara, \\ México;" "Federación Mexicana de Colegios de Ortopedia y Traumatología, A. C. Aguascalientes, México;"Sanatorio San Francisco \\ de Asís, Guadalajara, México; * Universidad de Guadalajara, Guadalajara, México.
}

\#¥Banuelos Benites R (Universidad Panamericana, Aguascalientes, México); Gutiérrez Mendoza I (Médico con práctica privada); Izaguirre A (Universidad Autónoma de Tamaulipas); Jiménez Ávila JM (Instituto Tecnológico y de Estudios Superiores de Monterrey, Campus Guadalajara, Guadalajara, Jalisco, México); López-Cervantes RE (Hospital San Francisco de Asís); López Estrada D (Hospital «Dr. Gustavo A Rovirosa Pérez», Secretaría de Salud de Tabasco); López-Mejía G (Hospital Christus Muguerza Saltillo); Martínez De León U (IMSS UMAE 14 Veracruz, Hospital de María, Veracruz); Martínez Islas L (Federación Mexicana de Colegios de Ortopedia y Traumatología, A.C.); Minguer Vargas S (Hospital Regional Presidente Juárez del ISSSTE); Quintero JE (Hospital Universitario San Jorge, Clínica Fracturas y Fracturas, Colombia); Rodríguez López J (Centro de Investigación y Docencia en Ciencias de la Salud de la Universidad Autónoma de Sinaloa); Salcido Reyna MV (Hospital General de Mexicali); Tabares Neyra H (Centro de Investigaciones en Longevidad, Envejecimiento y Salud (CITED)); Tafoya-Arreguin GA (Antiguo Hospital Civil de Guadalajara «Fray Antonio Alcalde»); Toledo-Infanson T (Hospital General Agua Prieta, Servicios de Salud Sonora); Vargas SL (IMSS Hospital No. 83, Morelia, Michoacán, México).

\section{Resumen}

Las soluciones para hacer frente la carga económica a causa de los traumatismos musculoesqueléticos en América Latina requieren no sólo recursos financieros e infraestructura de investigación, sino también la creación de capacidad de liderazgo para los cirujanos ortopédicos. Reconociendo el impacto que la capacitación para el desarrollo del liderazgo tiene en la mejora de los resultados para la atención de los pacientes y el desempeño de las organizaciones de atención médica, los cirujanos ortopédicos en América Latina podrían beneficiarse enormemente con estas oportunidades. Sin embargo, las oportunidades para asistir a los programas, particularmente en países con recursos más limitados, en gran medida no existen; la literatura que describe los programas de liderazgo se concentra en los países de ingresos altos (PIA), específicamente en América del Norte y Europa. Como resultado de esta discrepancia, la red, Asociación de Cirujanos Traumatólogos de las Américas (ACTUAR), desarrolló planes de estudio para un Programa de Desarrollo de Liderazgo (PDL) para cirujanos ortopédicos latinoamericanos. En este artículo se describe el desarrollo del PDL impartido a 40 cirujanos traumatólogos ortopédicos en el XXX Congreso Mexicano de la Federación Mexicana de Colegios de Ortopedia y Traumatología A.C. (FEMECOT) en Hermosillo, México, el 31 de octubre de 2019.

Palabras clave: Creación de capacidad de liderazgo, cirugía ortopédica, ortopedia y traumatología, desarrollo de liderazgo, curso de competencia no clínica.

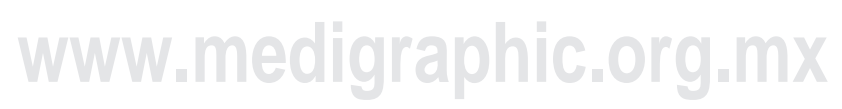

Correspondencia:

Theodore Miclau, MD

Zuckerberg San Francisco General Hospital

2550 23rd Street, Building 9, 2nd Floor,

San Francisco, CA, USA, 94110, Teléfono: 628-206-8812.

E-mail: theodore.miclau@ucsf.edu

Citar como: MacKechnie MC, Padilla RLG, De la Huerta F, López AL, Gómez AJM, Quintero HS, et al. Creación de capacidades para el desarrollo de liderazgo a los cirujanos ortopédicos en América Latina.

Recibido: 08-07-2021. Aceptado: 25-07-2021. Orthotips. 2021; 17 (4):211-216. https://dx.doi.org/10.35366/102219 


\begin{abstract}
Solutions to address the global burden of musculoskeletal trauma in Latin America require not only financial resources and clinical research infrastructure, but also non-clinical leadership capacity-building for orthopaedic surgeons. Recognizing the impact that leadership development training has on improving patient outcomes and performance of healthcare organizations, orthopaedic surgeons in Latin America could greatly benefit from these opportunities. Yet, formal training or opportunities to attend such programs, particularly in countries with limited resources, largely do not exist. Most of the literature describing leadership programs is concentrated on high-income countries (HICs), specifically in North America and Europe. As a result of this discrepancy, the network of orthopaedic trauma surgeons, Asociación de Cirujanos Traumatólogos de las Américas (ACTUAR), developed curricula for a novel Leadership Development Program (LDP) for Latin American orthopaedic surgeons. This paper describes the delivery of the one-day LDP delivered to forty orthopaedic trauma surgeons at the $30^{\text {th }}$ Annual Federación Mexicana de Colegios de Ortopedia y Traumatología (FEMECOT) Congress in Hermosillo, México on October 31, 2019.
\end{abstract}

Keywords: Building leadership capacity, orthopaedic surgery, orthopaedics and traumatology, leadership development, non-clinical competency course.

\section{Introducción}

Las condiciones de patologías traumáticas musculoesqueléticas contribuyen a aumentar la carga económica en todo el mundo. ${ }^{1}$ Según la Organización Mundial de la Salud (OMS), América Latina tiene las tasas más altas de mortalidad por accidentes de tráfico per cápita que cualquier otra región del mundo. ${ }^{2}$ América Latina cuenta con diversos sistemas de salud y distintos recursos según los países, ${ }^{3}$ y tiene una relativa ausencia de investigaciones específicas de la región que examinen la carga económica a causa de los traumatismos musculoesqueléticos. Esta falta de investigación procedente de América Latina contribuye a la escasez general de datos creíbles sobre las condiciones musculoesqueléticas en los países de ingresos bajos y medianos (PIBM). ${ }^{4}$ Las soluciones específicas de cada región para hacer frente a esta problemática requieren no sólo recursos financieros e infraestructura de investigación, sino también la creación de capacidad de liderazgo para los cirujanos ortopédicos. Reconociendo el impacto que la capacitación para el desarrollo del liderazgo tiene en la mejora de los resultados para la atención de los pacientes y el desempeño de las organizaciones de atención médica, los cirujanos ortopédicos en América Latina podrían beneficiarse enormemente con estas oportunidades. ${ }^{5-9}$

Las competencias de liderazgo no clínicas, como la inteligencia emocional, autoconciencia, comunicación eficaz, habilidades para resolver problemas, así como la capacidad de entrenar y proveer tutoría a otros son características comunes que se esperan en el campo de la cirugía ortopédica. Sin embargo, la capacitación formal o las oportunidades para asistir a dichos programas, particularmente en países con recursos más limitados, en gran medida no existen; ${ }^{10-12}$ la mayor parte de la literatura que describe los programas de liderazgo se concentra en los países de ingresos altos (PIA), específicamente en América del Norte y Europa. ${ }^{11}$ Como resultado de esta discrepancia, la Red Latinoamericana de Cirujanos Ortopédicos de Trauma, Asociación de Cirujanos Traumatólogos de las Américas (ACTUAR; www.actuarla.org), desarrolló planes de estudio para un novedoso Programa de Desarrollo de Liderazgo (PDL) para cirujanos ortopédicos latinoamericanos. El propósito de este curso es complementar las habilidades personales y profesionales del impulso de los cirujanos ortopédicos para liderar con la experiencia necesaria, y hacerlo de manera efectiva. EI PDL podría ser adaptado y utilizado como modelo por cirujanos ortopédicos locales en ambientes con recursos limitados.

En este artículo, se describe el desarrollo del PDL, impartido a cuarenta cirujanos traumatólogos ortopédicos en el XXX Congreso Mexicano de la Federación Mexicana de Colegio de Ortopedia y Traumatología A.C. (FEMECOT) en Hermosillo, Sonora, México, el 31 de octubre de 2019.

\section{Material y métodos}

\section{Antecedentes}

Existe una disparidad en el número de publicaciones científicas procedentes de América Latina, en particular dentro de la subespecialidad de traumatología; ${ }^{13}$ en efecto, sólo $1 \%$ de todos los artículos de investigación académica se publican en las principales revistas ortopédicas, y sólo $3.1 \%$ de los estudios latinoamericanos de trauma ortopédico están representados entre los PIBM. ${ }^{13,14}$ Esta deficiencia es considerada altamente significativa si se tiene en 
cuenta la importancia de la investigación producida localmente en el desarrollo de una infraestructura sanitaria eficaz. ${ }^{15,16}$ Las oportunidades de desarrollo profesional, como el desarrollo del liderazgo, son fundamentales para desarrollar vías de intercambio de conocimientos y creación de capacidades. ${ }^{17-19}$ Una de las soluciones es la creación de asociaciones multinacionales que promuevan los esfuerzos de colaboración en el trabajo de investigación y la infraestructura de liderazgo. Por lo tanto, en el 2017 se desarrolló la Asociación de Cirujanos Traumatólogos de las Américas (ACTUAR) con el fin de fomentar las alianzas y facilitar el trabajo en red entre el Norte-Sur Global. ${ }^{20}$ Desde su creación, ACTUAR ha crecido hasta contar con más de 150 miembros y se ha asociado con más de 20 sociedades académicas de toda la región. Con el objetivo general de aumentar la capacidad de investigación en América del Norte, del Sur y Central, ACTUAR trabaja para seguir desarrollando la infraestructura de cooperación con estos cirujanos como colaboradores, coinvestigadores y coautores.

Comprometerse y apoyar el desarrollo de la capacidad de liderazgo parece ser una estrategia eficaz para mejorar los resultados y el tratamiento de los pacientes. En la actualidad, existen pocas oportunidades de desarrollo de liderazgo para que los cirujanos ortopédicos de América Latina participen en cursos no clínicos y de competencias, lo cual limita su capacidad de mejorar sus habilidades como líderes. Dado que muchos miembros de ACTUAR desempeñan funciones de liderazgo formales e informales como miembros en juntas directivas de sociedades ortopédicas nacionales e internacionales, y como mentores de alumnos en ortopedia durante sus prácticas clínicas, la formación para el desarrollo del liderazgo podría tener un gran impacto en estos cirujanos-líderes, tanto personal como profesionalmente.

Como resultado, en 2019 los miembros de ACTUAR y los profesores de la Universidad de California, San Francisco (UCSF) y la Federación Mexicana de Colegios de Ortopedia y Traumatología, A.C. (FEMECOT) colaboraron en el diseño de un curso de liderazgo, desarrollado en un día, que se impartió en uno de los mayores congresos anuales de ortopedia en América Latina. Dada la relativa ausencia de modelos de programas de liderazgo para cirujanos ortopédicos, a los principales organizadores del curso se les encomendó la tarea de elaborar un plan de estudios adaptado a la cirugía ortopédica para que fuera pertinente y aplicable.

\section{Resultados}

\section{Curso de liderazgo}

El 31 de octubre de 2019, 40 cirujanos ortopédicos de tres países (Colombia, Cuba y México) asistieron al PDL de un día de duración en Hermosillo, Sonora, México, celebrado dentro del marco XXX Congreso Mexicano FEMECOT. Entre los asistentes se incluyeron cirujanos ortopédicos, seleccionados con base en su experiencia, funciones como líderes en sus sociedades ortopédicas profesionales o en sus consultas clínicas. Como preparación para el curso, los asistentes recibieron una encuesta de evaluación de necesidades para verificar su nivel de interés e importancia en los temas de liderazgo, los aceleradores y obstáculos percibidos para asistir a las oportunidades de desarrollo del liderazgo, así como información demográfica. ${ }^{21}$ Los temas de liderazgo identificados a partir de los resultados de la encuesta, guiaron a los organizadores en la selección del contenido del curso del PDL.

El programa del PDL consistió en múltiples métodos de enseñanza, como la didáctica, los pequeños grupos de trabajo y las actividades interactivas dentro del grupo (Apéndice 1), y se desarrolló de acuerdo con la regla «70-20-10» para fomentar la capacidad de liderazgo eficaz. ${ }^{22,23}$ Diez por ciento del plan de estudios consistió en instrucción formal y conceptos de aprendizaje en el aula, 20\% en debates y comentarios en grupos pequeños, y $70 \%$ en aprendizaje experimental y aplicación de sus nuevas habilidades a experiencias del mundo real. Esto también se logró a través de una actividad de grupo interactiva y la instrucción fue impartida por cirujanos-líderes locales con un interés y experiencia en áreas selectivas de desarrollo de liderazgo (Figura 1). El programa de los cursos se diseñó identificando los temas y tópicos clave a partir de revisiones bibliográficas centradas en América Latina, ${ }^{17,24-27}$ programas formales de liderazgo de las principales escuelas de negocios de Estados Unidos y recomendaciones solicitadas a expertos. El plan de estudios incluyó conceptos diseñados para impartir los principios fundamentales del desarrollo del liderazgo, en concreto, los elementos críticos del liderazgo, los estilos de aprendizaje personal, la comunicación, la dinámica de equipo y las actividades pedagógicas prácticas, incluido un estudio de caso y una actividad de grupo interactiva. Toda la instrucción del curso fue en el idioma español. 


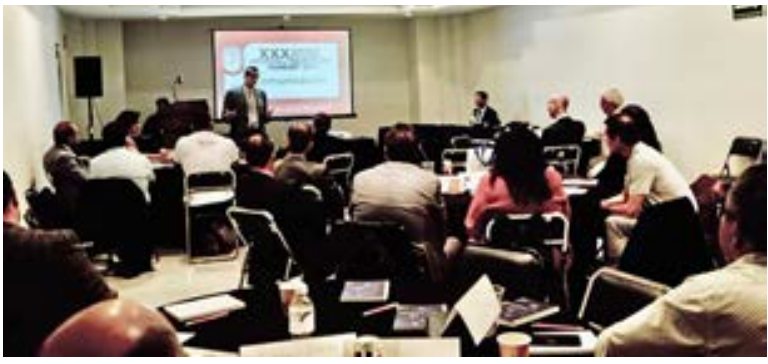

Figura 1: El Dr. Fernando de la Huerta de Guadalajara, México, realizó una presentación sobre comunicación efectiva en el Programa de Desarrollo de Liderazgo (PDL).

\section{Discusión}

\section{Direcciones de futuro}

EI PDL intensivo, presentado por profesores locales sobre los fundamentos del liderazgo, podría ser un método eficaz a largo plazo para apoyar la capacidad de desarrollo del liderazgo y, en última instancia, mejorar el tratamiento de la atención de los traumatismos musculoesqueléticos en diversas poblaciones de América Latina.

Las direcciones futuras podrían incluir: 1) la transición del PDL a un plan de estudios modular en línea para permitir una formación accesible y a su propio ritmo, así mismo un impacto amplificado con un alcance más amplio como cirujanos ortopédicos; 2) la adaptación del curso para otras subespecialidades 0 regiones geográficas, en particular en los PIBM que a menudo carecen de estas oportunidades de desarrollo profesional; 3) mantener un modelo sustentable mediante la inclusión de cirujanos-líderes locales como educadores con interés y experiencia en el liderazgo; 4) ampliar el PDL mediante la creación de una serie de cursos complementarios que se impartirán cada año en futuros congresos de la FEMECOT; y 5) evaluar y medir la retención de conocimientos y el impacto del curso mediante encuestas previas y posteriores a los conocimientos.

\section{Conclusiones}

En resumen, el PDL fue diseñado para apoyar y brindar nuevas oportunidades a los cirujanos ortopédicos en posiciones de liderazgo de alto nivel en toda América Latina que están preparados para impactar la especialidad hoy y en el futuro. Este tipo de curso podría seguir enriqueciendo el desarrollo de habilidades de liderazgo no clínicas de los cirujanos, aumentando su conocimiento de los conceptos y principios de liderazgo, facilitando la creación de redes y reforzar las asociaciones globales Norte-Sur. El objetivo de este curso fue desarrollar un modelo sostenible y eficaz, y dotar a los cirujanos ortopédicos de América Latina de las habilidades de liderazgo necesarias y, en última instancia, hacer avanzar la atención musculoesquelética de alta calidad. Aunque la formación para el desarrollo del liderazgo en medicina tiene muchos beneficios claros, es necesario seguir investigando el impacto de un PDL basado en la evidencia específicamente para cada región, con el fin de orientar y mejorar los planes de estudio de futuros cursos.

\section{Agradecimientos}

Agradecemos el apoyo de la Federación Mexicana de Colegios de Ortopedia y Traumatología, A.C. (FEMECOT) y la fundación Médica Wyss.

\section{Referencias}

1. Murray CJ, Vos T, Lozano R, Naghavi M, Flaxman AD, Michaud $\mathrm{C}$, et al. Disability-adjusted life years (DALYs) for 291 diseases and injuries in 21 regions, 1990-2010: a systematic analysis for the Global Burden of Disease Study 2010. Lancet. 2012; 380 (9859): 2197-2223.

2. World Health Organization. Global status report on road safety: time for action [Online]. Geneva: World Health Organization; 2009. [Accessed December 19, 2020] Available in: https://apps. who.int/iris/bitstream/handle/10665/44122/9789241563840 eng.pdf;jsessionid=52695470F483BBEB25BFC5685C7FDC 04? sequence $=1$

3. Padilla Rojas LG, López Cervantes RE, Pérez Atanasio JM, Sánchez MM, Gómez Acevedo JM, Kodima KE. Latin America Trauma Systems - Mexico and Brazil. OTA Int. 2019; 2 (S1): e020.

4. Joshipura M, Gosselin RA. Surgical burden of musculoskeletal conditions in low- and middle-income countries. World J Surg. 2020; 44 (4): 1026-1032. doi: 10.1007/s00268-018-4790-8.

5. Lerman C, Jameson JL. Leadership development in medicine. N Engl J Med. 2018; 378 (20): 1862-1863.

6. Frenk J, Chen L, Bhutta ZA, Cohen J, Cris N, Evans T, et al. Health professionals for a new century: transforming education to strengthen health systems in an interdependent world. Lancet. 2010; 376: 1923-1958.

7. Reinertsen JL. Physicians as leaders in the improvement of health care systems. Ann Intern Med. 1998; 128 (10): 833-838.

8. Rotenstein LS, Sadun R, Jena AB. Why doctors need leadership training. Harv Bus Rev. 2018; 17.

9. Baker GR, Macintosh-Murray A, Porcellato C, et al. High performing health care systems: delivering quality by design. In: Longwoods; 2008.

10. Lobas JG. Leadership in academic medicine: capabilities and conditions for organizational success. Am J Med. 2006; 119 (7): 617-621. doi: 10.1016/j.amjmed.2006.04.005. 
11. Frich JC, Brewster AM, Cherlin EJ, Bradley EH. Leadership development programs for physicians: a systematic review. J Gen Intern Med. 2014; 30 (5): 656-674. doi: 10.1007/s11606014-3141-1.

12. Alpert JS. Leadership in Academic Medicine. Am J Med. 2010; 123: 1071-1072.

13. Challa S, Wu HH, Cunningham BP, Liu M, Patel K, Shearer DW, et al. Orthopaedic trauma in the developing world: where are the gaps in research and what can be done? J Orthop Trauma. 2018; 32 Suppl 7: S43-S46. doi: 10.1097/BOT.0000000000001293.

14. Urrutia J, Zamora T, Prada C. The fifty most cited Latin American articles in the orthopaedic literature. Int Orthop. 2014; 38 (8): 1723-1729.

15. Nuyens $Y$. No development without research: a challenge for research capacity strengthening. Geneva, Switzerland: Global Forum for Health Research; 2005. Available in: https://www.files.ethz.ch/isn/128224/2005-ResearchReportNoDevelopment.pdf

16. Minja H, Nsanzabana C, Maure C, Hoffmann A, Rumisha $\mathrm{S}$, Ogundahunsi $\mathrm{O}$, et al. Impact of health research capacity strengthening in low- and middle-income countries: the case of WHO/TDR programmes. PLoS Negl Trop Dis. 2011; 5 (10): e1351.

17. Miclau TA, Chomsky-Higgins K, Ceballos A, Balmaseda R, Morshed S, Bhandari M, et al. Building surgical research capacity globally: efficacy of a clinical research course for surgeons in low-resource settings. Front Educ. 2017; 2: 57. doi: 10.3389/feduc.2017.00057.

18. Prado AM, Pearson AA, Bertelsen NS. Management training in global health education: a Health Innovation Fellowship training program to bring healthcare to low-income communities in Central America. Glob Health Action. 2018; 11 (1): 1408359. doi: 10.1080/16549716.2017.1408359.

19. Hoppe B, Reinelt $C$. Social network analysis and the evaluation of leadership networks. Leadersh Q. 2010; 21: 600-619.

20. Miclau T, MacKechnie MC, Shearer DW; ACTUAR group. Asociación de Cirujanos Traumatólogos de las Américas:
Development of a Latin American Research Consortium. J Orthop Trauma. 2018; 32 Suppl 7: S8-S11. doi: 10.1097/ BOT.0000000000001302.

21. MacKechnie MC, MacKechnie MA, van Lieshout EMM, et al. Leadership development for orthopaedic surgeons in Latin America: opportunities for and barriers to skill acquisition. OTA Int. 2021. In Press.

22. Rosch DM, Jenkins DM. What do we know about formal leadership courses and their effects? New Dir Stud Leadersh. 2020; 2020 (168): 31-41.

23. Eichinger B, Lombardo M. Center for Creative Leadership: lessons of experience. [Accessed June 20, 2021] Available in: https://www.ccl.org/articles/leading-effectively-articles/7020-10-rule/

24. Chomsky-Higgins K, Miclau TA, Mackechnie MC, Aguilar D, Avila JR, Dos Reis FB, et al. Barriers to clinical research in Latin America. Front Public Health. 2017; 5: 57. doi: 10.3389/ fpubh.2017.00057.

25. Wu E, Singh M, Le PV, Dandu M, Duderstadt K, Brock T, et al. Global Health Bootcamp: an innovative interprofessional course for clinicians dedicated to equitable Global Health Care Delivery. Ann Glob Health. 2015; 81 (1): 60-61.

26. Steinert $Y$, Naismith L, Mann K. Faculty development initiatives designed to promote leadership in medical education. ABEME systematic review. Med Teach. 2012; 34 (6): 483-503.

27. Mano MS, Gomes R, Werutsky G, Barrios CH, Marta GN, Villarreal-Garza $\mathrm{C}$, et al. Cross-cultural validity study of a medical education leadership competencies instrument in Latin American physicians: a multinational study. J Glob Oncol. 2019; 5: 1-9. doi: 10.1200/JGO.19.00243.

\section{Conflicto de intereses}

No hay conflicto de intereses. 
Apéndice 1:

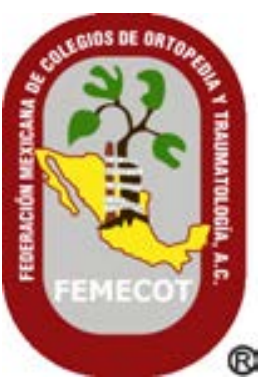

\section{Leadership Development Program for Orthopaedic Surgeons in Latin America}

Thursday October 31, 2019

8:00am-3:30pm

\section{AGENDA}

8:00am-8:30am Introductions/Welcome

8:30am-10:30am Leadership: Why, How, and What?

10:30am-11:00am Break

11:00am-12:30pm Leadership Development

- Leadership Qualities: Critical Elements

- Personal Learning Styles

- Communication

12:30pm-1:30pm Workshop: Team Dynamics

1:30pm-2:30pm What Have We Learned?

3:30pm Adjourn

ACTUAR 